\title{
Conductor Temperature Monitoring under different current using FBG Sensor Jing Yin $^{1}$, Dehua Shu ${ }^{1}$, Mingshun Jiang ${ }^{\mathrm{a} 2}$, Yaozhang Sai ${ }^{2}$, Qingmei Sui ${ }^{2}$ \\ ${ }^{1}$ Aerospace Electronic Technology Research institute of Shandong Province \\ ${ }^{2}$ School of Control science \& Engineering, Shandong University \\ aemail: sdujiangmingshun@163.com
}

Keywords: Temperature monitoring; FBG; Fusing state

\begin{abstract}
This paper studies the relationship between the fusing characteristics of copper wire and the overload current. Based on a physical model, the fusing curve for the copper wire is obtained. At the same time, fiber Bragg grating (FBG) is used to monitor the temperature change of aerospace conductor. According to the experimental result, FBG can detect temperature in time, and current and fusing state can be predicted by the temperature and the rate of temperature rise. So FBG is suitable for the aerospace wire fault monitoring.
\end{abstract}

\section{Introduction}

In this paper a new optimal design of soccer robot control system which is based on mechanical analyses and calculations on the pressure and transmutation states of chip kick mechanics, this new control system with high precision for speed control and high dynamic quality.

In aerospace equipment, wires will damage under electrical-over-stress. Wire burned problems are common problems in the world aerospace field. NASA has published research report on wire burned, and has funded the study of wire fusing features of flight test program[1,2].

At present, metal wire fusing physical model can better characterize fusing characteristic of uniform diameter of wire in atmospheric environment or vacuum environment. In actual situation, conductor contains many metal wire, and has Teflon coating. New physical model and test must be introduced[3].

\section{Conductor Temperature Characteristic Research}

Generally, wire heat conduction is expressed as Q1. The heat capacity of heat is expressed as Q2. Heat power provided by electricity is expressed as Q.

(1) Calculation of Heat Transfer Loss

According to the Fourier conduction law: $Q_{1}^{\prime}=-k \nabla T$

where $k$ is thermal conductivity. $\nabla T=\frac{d T}{d x}$ is temperature gradient.

According to Wiedemann-Franz law, the relationship of the metal thermal conductivity $k$ and electrical conductivity $\sigma$ is as follows:

$$
\frac{k}{\sigma T}=\frac{\pi^{2}}{3}\left(\frac{K}{q}\right)^{2}
$$

$K$ is the boltzmann constant. $q$ is free electron charge. The relationship of electrical conductivity $\sigma$ and temperature is as follows:

$$
\sigma=\frac{1}{\rho}=\frac{1}{\rho_{0}(1+\alpha t)}
$$

$\rho$ is the electrical resistivity of metal. $\rho_{0}$ is the electrical resistivity when temperature is $20{ }^{\circ} \mathrm{C} . \alpha$ is the temperature coefficient of resistance.

$$
\text { Eq (1) can be rewirtten as: } Q d x=k d T=\left(\frac{K}{q}\right)^{2} \frac{\pi^{2}(273+t) d t}{3 \rho_{0} \alpha[(1 / \alpha)+t]}
$$


Eq (5) is treated by integral. The wire transfer heat can be expressed as:

$Q_{1}^{\prime}=\frac{K^{2} d^{2} \pi^{3} t^{\prime}}{3 q^{2} \rho_{0} \alpha l}\left[\left(273-\frac{1}{\alpha}\right) \times \ln \left(\frac{\frac{1}{\alpha}+t}{\frac{1}{\alpha}+t_{0}}\right)+\left(t-t_{0}\right)\right]$

$t^{\prime}$ is time. $\mathrm{t}$ is centigrade temperature when metal wire fusing. $t_{0}$ is centigrade temperature of the environment. $\quad l$ is the length of metal wire[4].

(2) The Calculation of Heat Conduction of Wire in Air

The length of wire is $l$, the infinitesimal length is $d x$ in any position. The temperature is $T=\nabla T \cdot x$. Assuming that temperature is $T_{0}$. According to Eq.(1),

the radial temperature gradient $: \nabla T^{\prime}=\frac{d T}{d r}=\frac{T-T_{0}}{l}=\frac{\nabla T x-T_{0}}{l}$

There is :

$Q_{1}^{\prime \prime}=2 \int d Q=\frac{2 k_{q} \pi d t^{\prime}}{l} \int_{0}^{\frac{1}{2}}\left(\nabla T x-T_{0}\right) d x=k_{q} \pi d t^{\prime}\left(\frac{\nabla T l}{4}-T_{0}\right)$

$k_{q}$ is the heat transfer coefficient of air. Heat of wire thermal conduction losses is[5]:

$Q_{1}=Q_{1}^{\prime}+Q_{1}^{\prime \prime}$

(3) The heat capacity and heat of wire fuse

The specific heat capacity of metal wire is $c . \rho^{\prime}$ is the density. Because of $d m=\rho^{\prime} d V=\frac{\rho^{\prime} \pi d^{2}}{4} d x$ and $d Q=c d m\left(T-T_{0}\right)=c\left(\nabla T x-T_{0}\right) \frac{\rho \pi d^{2}}{4} d x$, there is:

$Q_{2}=2 \int d Q=\frac{c \rho^{\prime} \pi d^{2}}{2} \int_{0}^{\frac{l}{2}}\left(\nabla T x-T_{0}\right) d x=c \rho^{\prime} \pi d^{2}\left(\frac{\nabla T l}{4}-T_{0}\right)$

The heat of wire fuse is :

$$
Q_{3}=m k^{\prime}=\frac{\rho^{\prime} \pi d^{2}}{4} \xi k^{\prime}
$$

$k$ is the heat capacity of metal wire fuse. $\xi$ is the length of the fuse.

(4) The Heat Provided by Power

Due to the resistance of the wire increases with temperature rise, $R=f(t)$. The temperature coefficient of metal wire is $\alpha$. The resistance of metal wire is[6]:

$R(t)=2 \int d R=\frac{4 \rho_{0}}{\pi d^{2}}\left[1+\alpha\left(\frac{\nabla T}{4} l-273\right)\right]$

The heat provided by power is $Q=Q_{1}+Q_{2}+Q_{3}$. So the relationship of current and time is:

$i_{d}=\frac{I}{S}=\sqrt{\frac{Q_{1}+Q_{2}+Q_{2}}{R\left(t^{\prime}\right) t^{\prime}}} \cdot \frac{1}{\pi d^{2}}$

\section{Temperature Monitoring Experiment}

(1) Experimental System

19 silver-plated copper wire wrapped around conductor is used in this experiment. Teflon layer is used to wrap conductor. The rated current of conductor is $2 \mathrm{~A}$. The length of Conductor is $500 \mathrm{~mm}$, and the diameter is $0.12 \mathrm{~mm}$. The experimental system is shown on figure 1 . The system includes of FBG, platinum resistance temperature sensor (PRTS), conductor, FBG demodulator, platinum resistance temperature system and constant current source. During the experiment, PRTS and FBG is bonded on the conductor by high-temperature plastic. Experimental environment temperature is $25^{\circ} \mathrm{C}$. 


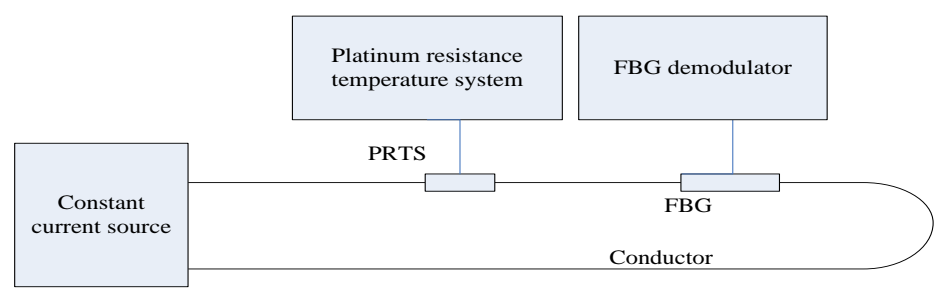

Fig 1. Experimental system

(2) Experimental Method

The temperature values of PRTS and FBG are recorded when there are 2A, 6A, 8A, 10A, 12A, 16A currents in conductor, respectively. The temperature values of PRTS and FBG are contrasted and discrete data are treated by use the binomial fitting method. The result is shown on Fig 2. According to fitted curve, the temperature curves of FBG and PRTS are consistent. According to temperature values, FBG has higher sensitivity. So FBG can completely replace the traditional temperature sensors.

According to fitting curve of FBG, current is greater, the growth of temperature is faster. Because the fusing temperature of conductor is $250^{\circ} \mathrm{C}$, the fusing current of conductor is $25 \mathrm{~A}$ according to fitting formula.

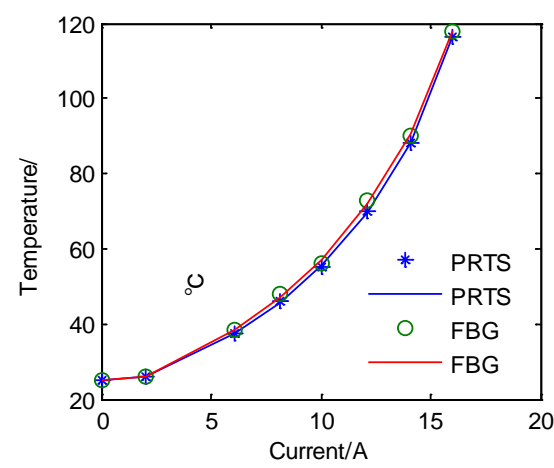

Fig. 2 The temperature curves of FBG and PRTS

After current increase, there is 5 minutes to make temperature stability. The whole experimental process is completely recorded. The result is shown on Fig 3. The temperature difference detected by using FBG is no more than $2^{\circ} \mathrm{C}$. Current is greater, the temperature rise is faster. The temperature rise can be expressed by the slope of temperature rise. The slope is shown on Fig 4 . When current is $18 \mathrm{~A}$, the rate of temperature rise is $0.96^{\circ} \mathrm{C} / \mathrm{s}$. According to fitting curve, when current is $25 \mathrm{~A}$ which is fusing current, the rate of temperature rise is $1.69^{\circ} \mathrm{C} / \mathrm{s}$. When the temperature fell sharply, conductor can be considered to be fused.

Bundle conductor has 6 conductors. 2A, 6A, 10A currents are separately loaded in every conductor. After current increase, there is 5 minutes to make temperature stability. The whole experimental process is completely recorded. The result is shown on Fig 5. When current is 10A, the temperature is $119^{\circ} \mathrm{C}$. The rate of temperature rise is shown on Fig 6. The more the number of conductors are, the faster the rate of the temperature rise is. For example, when current is $10 \mathrm{~A}$, the rate of temperature rise of bundle conductor is $0.72^{\circ} \mathrm{C} / \mathrm{s}$. The rate of temperature rise of single conductor is only $0.344^{\circ} \mathrm{C} / \mathrm{s}$.

\section{Conclusion}

According to the physical model of wire, there is a direct relationship between current and wire radiation heat loss. Heat responses the change of temperature. According to the temperature of wire and the rate of temperature rise and fell, the current and fusing state can be predicted.

In this paper, high reliability and high sensitivity of FBG are verified. Based on the FBG temperature system, the current of conductor and bundle conductor can be judged by the detected temperature values. At the same time, the access and short state of conductor can be predicted. So 
FBG is suitable for the aerospace wire fault monitoring.

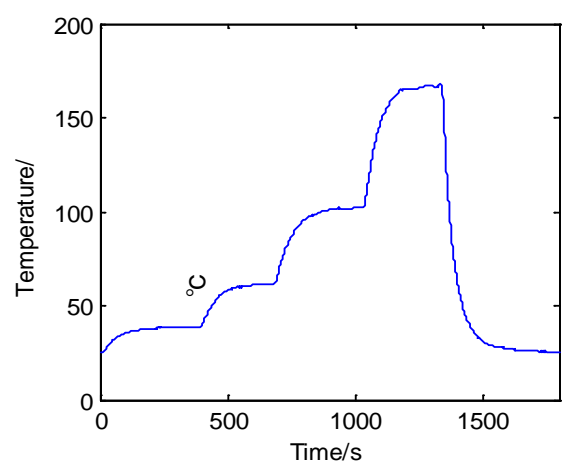

Fig.3 Continuous loading current

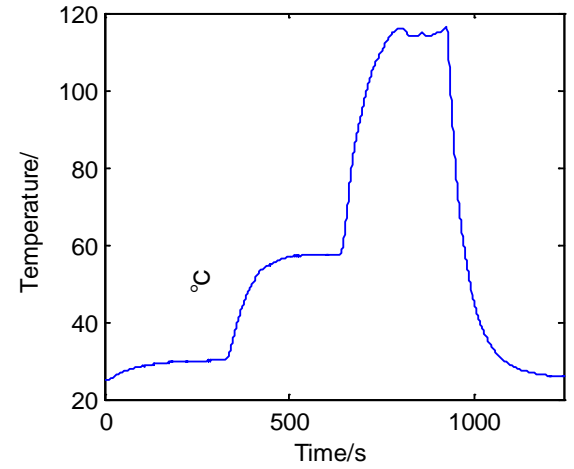

Fig.5 Continuous loading current

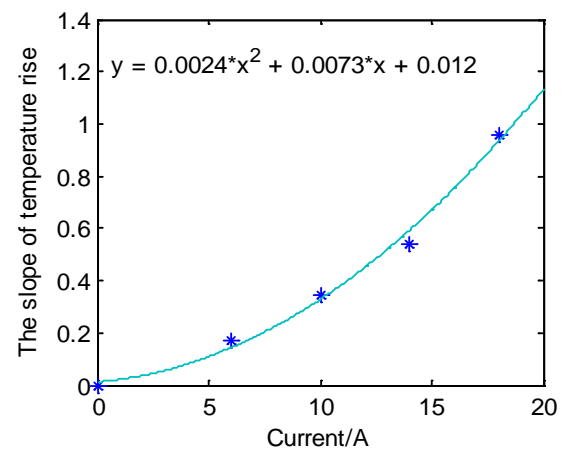

Fig.4 The rate of temperature rise

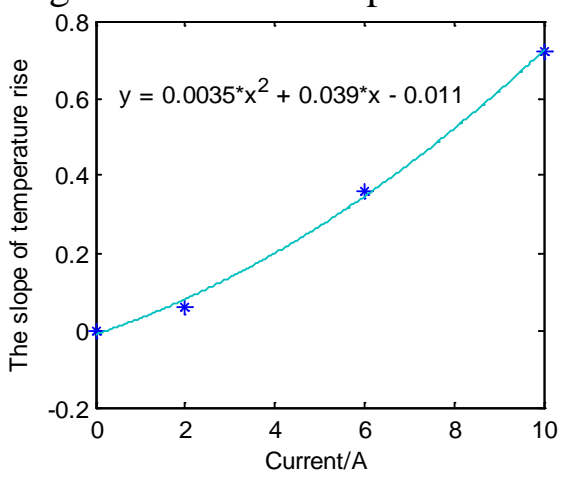

Fig.6 The rate of temperature rise

\section{References}

[1] Kong WJ, Wang BR, Lao SQ. Study on fire precursor of wire insulation in low-pressure environments. Journal of Engineering Thermophysics. 2007.28:1047-1049.

[2] Chen LF, Xin Z, Kong WJ, et. al. Functional simulations of the fire precursor of the wire insulation in quiescent microgravity environment. Chin. J. Space Sci..2006.26:235-240.

[3] Kong WJ, Wang BR, Lao SQ, et al. Study on fire initiation of wire insulations on board the satellite.Chin. J. Space Sci.2008.28:28-32.

[4] Chen Y, Zhang W. The fusing characteristics of copper wire in space applications. Spacecraft Environment Engineering.2013.30:407-410.

[5] Wang MZ, Wang YH, Zhou YG. On property of fusing of metal wire. Journal of Sichuan University of Science \& Engineering. 2008.21:12-15. 